\title{
Lesión necrotizante de la línea media con fístula oronasal causada por inhalación de cocaína
}

\section{Cocaine-induced midline destructive lesion and oronasal fistula}

\author{
Crovetto Martínez R*, Whyte Orozco J**, Cisneros AI***, \\ Crovetto de la Torre $M A * * * *$
}

\section{RESUMEN}

Los autores realizan una revisión bibliográfica de las perforaciones palatinas asociadas a lesiones necrotizantes de la línea media por inhalación de cocaína y, aportan un caso clínico propio con fístula oronasal. La frecuencia de las perforaciones palatinas asociadas a las lesiones necrotizantes de la línea media por inhalación de cocaína es muy superior a las encontradas en la granulomatosis de Wegener. La presencia de una perforación palatina es también un dato sugestivo de linfoma extranodal. La clave diagnóstica de todas las lesiones necrotizantes de la línea media es histopatológica pero obtener un diagnóstico inequívoco es más difícil de obtener de lo que podría suponerse.

Palabras clave: Lesiones necrotizantes de la línea media, cocaína, fístula oronasal.

\section{SUMMARY}

The authors perform a literature review of palatal fistulas present in midline necrotizing nasal lesions due to inhalation of cocaine and present a case report. The frequency of palatal fistulas associated with midline necrotizing lesions by inhalation of cocaine is superior to tose found in Wegener's granulomatosis. The presence of a palatal fistula is also suggestive of a extranodal lymphoma. Histopathology is the key to the differential diagnosis but getting an unequivocal diagnosis is more difficult to obtain than might be supposed.

Key words: Midline necrotizing nasal lesions, cocaine, palatal fistula.

Fecha de recepción: 1 de abril de 2013.

Aceptado para publicación: 28 de abril de 2013.

* Profesor interino del Departamento de Estomatología de la Facultad de Medicina y Odontología de la UPV/EHU.

** Profesor Titular. Departamento de Anatomía e Histología de la Facultad de Medicina de la Universidad de Zaragoza.

*** Profesor asociado. Departamento de Anatomía e Histología de la Facultad de Medicina de la Universidad de Zaragoza.

**** Profesor Titular. Jefe de Sección de Otorrinolaringología del Hospital Universitario de Basurto. UPV/ EHU.

Crovetto Martínez R, Whyte Orozco J, Cisneros AI, Crovetto de la Torre MA. Lesión necrotizante de la línea media con fístula oronasal causada por inhalación de cocaína Av. Odontoestomatol 2014; 30 (2): 63-67. 


\section{INTRODUCCIÓN}

La denominación "granuloma letal de la línea media" ha agrupado clásicamente una serie de procesos de distinta etiología que comportan la destrucción de los tejidos mucosos y osteocartilaginosos de la línea media facial y nasosinusal. Estos procesos son el linfoma y la granulomatosis de Wegener. Durante un tiempo también se incluía dentro de los procesos destructivos de la línea media a la Reticulosis Polimorfa (o reticulosis maligna mediofacial), pero ulteriormente se apreció que estas últimas correspondían a Linfomas de células NK/T (1). Los avances terapéuticos tanto en el tratamiento de la granulomatosis de Wegener como en los linfomas permiten curar con éxito a muchos de los pacientes afectados, por lo que la denominación genérica de "granuloma letal de la línea media" ha quedado sustituido por otras más acordes con la realidad, como "lesiones necrotizantes de la línea media" (2). De otro lado, el incremento de la adicción a la inhalación nasal de cocaína ha supuesto la aparición de un nuevo proceso capaz de provocar lesiones destructivas osteocartilaginosas mediofaciales (CIMDL: cocaine-induced midline destructive lesions), muy similares a las encontradas en los procesos causantes de "lesiones necrotizante de la línea media" $(3,4)$, lo que supone el advenimiento de una nueva entidad a considerar en el diagnóstico diferencial de tales procesos. Además deben considerarse dentro de las lesiones necrotizantes de la línea media otros procesos, como la sarcoidosis, la tuberculosis, la lepra, el lupus y otras infecciones muy infrecuentes (2).

El objetivo de este trabajo es realizar una revisión de las perforaciones palatinas asociadas a la inhalación nasal de cocaína en la literatura especializada y, mediante la aportación de un caso clínico propio, considerar el valor de dichas perforaciones en el diagnóstico diferencial con otras lesiones necrotizantes de la línea media, especialmente con la granulomatosis de Wegener.

\section{MATERIAL}

Revisión general de la bibliográfica en la literatura médica, usando la base PubMed, introduciendo los términos: Palate perforation, palatal fistula, hard palate destruction, oro-nasal communication, midline necrotizing nasal lesions, central facial destructive granuloma, midline necrosis, Wegener granulomatosis, cocaine-induced mildline destructive lesions.

\section{CASO CLÍNICO}

Mujer de 45 años sin antecedentes patológicos de interés que acudió a la consulta de Otorrinolaringología por presentar obstrucción nasal, formación de costras nasales y rinorrea sanguinolenta. Negó con vehemencia cualquier hábito tóxico, salvo el del tabaco. La exploración otorrinolaringológica reveló una gran perforación anterior del tabique nasal, asociada a la presencia de costras nasales adherentes y fétidas. En cavidad oral se apreciaba, en el centro del paladar duro, una perforación palatina tapada por las abundantes costras nasales.

La analítica (hematimetría completa con recuento y fórmula, glucemia, urea, ácido úrico, creatinina, filtrado glomerular, transaminasas, electrolitos, ácido fólico, vitamina B12, hormonas tiroideas, proteínas totales y proteinograma, PCR, factor reumatoide) fue normal. Las cifras de Inmunoglobulinas IgG $(559 \mathrm{mg} /$ Dl) estaban ligeramente deprimida y la de IgM (593 $\mathrm{mg} /$ dl) duplicaban el máximo valor normal. La VSG fue de $25 \mathrm{~mm}$ la primera hora. La serología HIV, hepatitis C, lues, brucella y borrelia fue negativa. La paciente, vacunada de hepatitis $B$, mostraba valores de anticuerpos de protección adecuados. Fueron también negativos los anticuerpos antinucleares, anti ds-DNA, y anticardiolipina IGG e IGM. Los estudios ANCA incluyeron dos valores: El PR3-ANCA $(0,6 \mathrm{UI} / \mathrm{ml})$ y el $\mathrm{MPO}$ ANCA $(<0,01 \mathrm{UI} / \mathrm{ml})$, ambos normales. Los análisis bioquímico y bacteriológico de orina también fueron normales. La radiografía simple de tórax no mostró ninguna alteración. Los cultivos bacteriológicos, micóticos y específicos de TBC fueron todos ellos negativos, salvo crecimientos de flora bacteriana mixta.

Las biopsias de tejido nasal (tres en total) fueron informadas, sucesivamente, como:

1. Proceso inflamatorio crónico con abundancia de linfocitos maduros, células plasmáticas e histiocitos.

2. Proceso inflamatorio crónico rico en células plasmáticas. 
3. Escaras fibrinoleucocitarias e inflamación crónica, con ausencia de vasculitis necrotizante.

Finalmente, la paciente, ya separada de la pareja que la acompañaba previamente, reconoció su adicción a la inhalación de cocaína, hábito que prometió abandonar (seis meses después de la primera consulta). Se procedió a la limpieza mecánica de las costras nasales, enfatizando los lavados con suero fisiológico y la toma diaria de comprimidos de trimetoprimsulfametoxazol (Septrim forte cada 12 horas) lo que motivo la desaparición progresiva de las costras nasales, aumentando tanto la rinolalia abierta como la regurgitación oronasal de alimento (figura 1). En la tomografía computarizada se observan con nitidez las destrucciones mediofaciales con afectación incluida del paladar óseo (figura 2). Se optó por cerrar dicha comunicación mediante tratamiento protésico, con buen resultado inicial. Dos años después de su aplicación variamos la prótesis aplicada por otra de menor tamaño que es más confortable pero que causa irritación de la mucosa palatina (figura 1).

Llama la atención que, aún años después de abandonado el hábito cocainómano la interrupción del tratamiento con trimetoprim-sulfametoxazol, se asocia a una recurrencia de las costras nasales, a pesar de los repetidos lavados nasales prescritos. Relacionamos el hecho con la presencia de una de una rinitis atrófica secuelar.

\section{RESULTADOS Y DISCUSIÓN}

La cocaína es una droga derivada de la hoja de coca que tiene un efecto anestésico local y estimulante del sistema nervioso central. Actúa mediante la alteración de la recaptación de tres neurotransmisores (norepinefrina, serotonina y dopamina). La cocaína tiene capacidad adictiva por que modula las respuestas de gratificación y motivación en el sistema mesolímbico (vía dopaminérgica) (5). Localmente, causa vasoconstricción. En las fosas nasales, su uso crónico puede causar necrosis mucosa y osteocartilaginosa.

El CIMDL comienza con la destrucción de la mucosa y el tabique nasal cartilaginoso, progresando luego

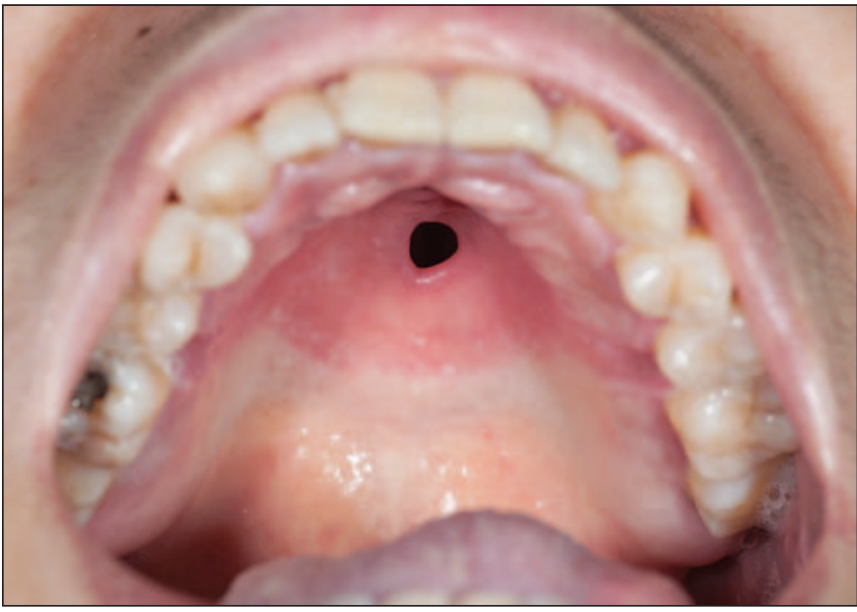

Fig. 1. Perforación medial del paladar duro que establece una comunicación oronasal. Nótese la hiperemia que afecta a parte del paladar y que corresponde a la presión ejercida por la prótesis obturadora.

al tabique óseo, cornetes, senos e incluso faringe. La destrucción puede asimismo afectar al paladar, provocando comunicaciones oronasales, con las consiguientes manifestaciones clínicas: rinolalia abierta y regurgitación alimentaria a las fosas nasales (6).

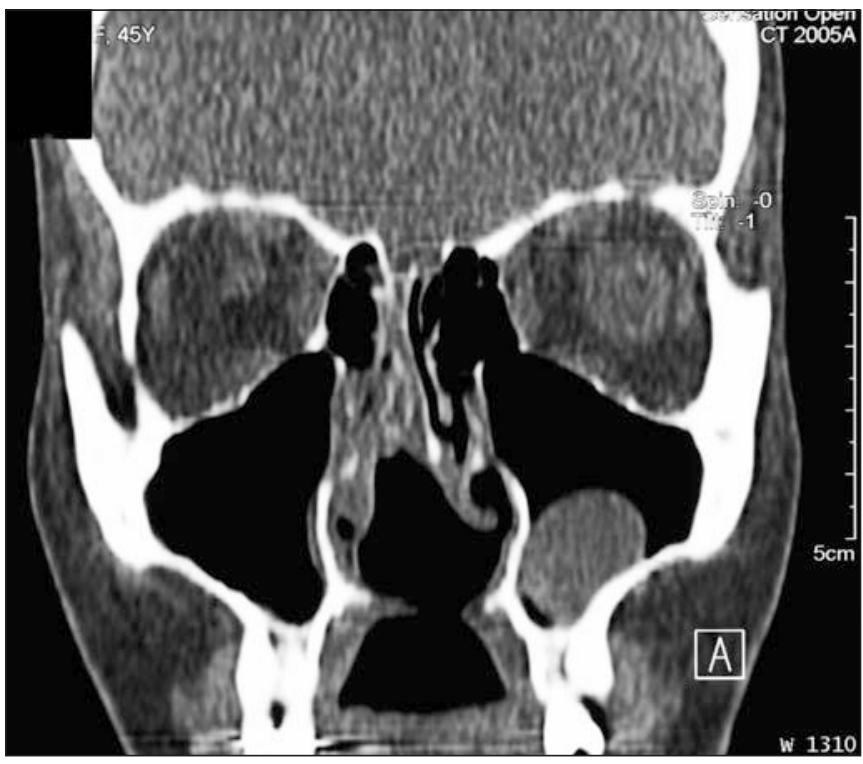

Fig. 2. Corte coronal obtenida median tomografía computarizada, donde se observa la ausencia de septo nasal y parcial desaparición de los cornetes. Hay, además, un quiste de retención en el seno maxilar izquierdo y una ocupación del etmoides anterior. Nótese la perforación palatina que establece una comunicación oronasal. 
El CIMDL plantea un diagnóstico diferencial con otras "lesiones necrotizantes de la línea media" pero muy especialmente con la granulomatosis de Wegener, cuyo comportamiento clínico mediofacial es similar, hasta el punto de que algunos autores reconocen haber tratado con medicación propia del Wegener a pacientes afectos de CIMDL (3). Las razones que dificultan el diagnóstico diferencial entre estos procesos son variadas. Así, muchos de los pacientes afectos de CIMDL niegan su adicción a la cocaína de forma insistente, durante meses o años en muchos casos, tal y como sucedió en el caso que presentamos, lo que dificulta el diagnóstico etiológico. De otro lado, aunque la clínica asociada al Wegener suele incluir manifestaciones del árbol respiratorio superior e inferior (nasosinusal, ótico, laríngeo, pulmonar), renales (glomerulonefritis), y sistémicas (7), hay formas de afectación localizada a una sola región (nasosinusal por ejemplo) (8-10), lo que la hace clínicamente indistinguible de la CIMDL. El diagnóstico diferencial es histopatológico demostrando lesiones granulomatosas y vasculíticas en el Wegener, pero resulta que la abundancia de costras y necrosis mediofaciales hace que los resultados sean muchas veces no concluyentes, al no encontrar el patólogo suficiente tejido útil para estudio, lo que obliga a repetir las biopsias con mucha frecuencia, siendo esta consideración cierta para todos los estudios histopatológicos de sujetos con lesiones necrotizantes de la línea media (2). Respecto al valor diagnóstico de los ANCA, la otra "piedra angular" del diagnóstico clásico de la granulomatosis del Wegener, encontramos que esos anticuerpos pueden estar elevados en la CIMDL (11). Precisamente la elevación de los ANCA en los pacientes con CIMDL permite sospechar una base inmune del mismo, en el sentido que sujetos predispuestos a este tipo de alteraciones podrían desencadenar la reacción autoinmune estimulados por el efecto irritante de la cocaína (9). De otro lado, y para complicar más las cosas, los títulos de ANCA pueden ser normales en las formas localizadas de granulomatosis de Wegener (2).

En el caso que presentamos, la paciente presentó valores normales de ANCA, lo que tuvo, a la luz de lo señalado, un valor diagnóstico relativo. En ninguna de las tres biopsias realizadas se pudo demostrar granulomatosis o vasculitis. No se pudie- ron demostrar tampoco compromisos pulmonares, ni renales, ni sistémicos. El diagnóstico de CIMDL se realizó por exclusión, y finalmente fue confirmado por la aceptación de la paciente de su hábito cocainómano.

Aunque la ulceración palatina no es excepcional en la granulomatosis de Wegener $(8,12)$, el establecimiento de una comunicación oronasal si que lo es, presentándose como casos aislados en la literatura (13). Así, ni Vega-Braga et al. (10) en una revisión de 17 casos, ni Cannady et al. (14) en una revisión de 120 casos refieren una sola perforación palatina (0 de 137 casos revisados). Sin embargo, en la CIMDL la comunicación oronasal es más frecuente cómo queda patente en la literatura. Así, Weistreich et al. (2) presentan 5 casos de perforación palatina de 7 pacientes afectados de CIMDL y recientemente Silvestre (15) presenta 4 casos más. En una reciente revisión de la literatura especializada se encuentran 36 casos de perforación palatina asociada al CIMDL de las que un $77,7 \%$ se localizan en el paladar duro y el resto en el blando o en ambos (6).

Debe considerarse, no obstante, que los linfomas de la línea media si que causan destrucciones palatinas y comunicaciones oronasales (2), por lo que su presencia plantea un diagnóstico diferencial fundamental con la CIMDL. Una vez más, es el estudio histopatológico lo que permite hacer un diagnóstico pero las dificultades diagnósticas para hacerlo no son pocas, resultando muy conveniente el concurso de patólogos entrenados en estos procesos de difícil diagnóstico diferencial.

\section{CONCLUSIÓN}

En presencia de lesiones necróticas en la línea media, con biopsias nasales no concluyentes, la presencia asociada de una perforación palatina, es más propia de CIMDL o de linfoma extranodal que de granulomatosis de Wegener.

No obstante la anamnesis exigente, descartando o confirmando el hábito cocainómano, el estado del paladar, el resultado del test de ANCA y el estudio de clínica sistémica asociada al Wegener son la base del diagnóstico provisional, hasta que se obtenga un 
diagnóstico definitivo basado en criterios histopatológicos, lo cual requiere paciencia y frecuentes repeticiones de biopsia, además de la colaboración de un patólogo entrenado en estos procesos.

\section{BIBLIOGRAFÍA}

1. Ishida F, Kwong YL. Diagnosis and management of natural killer-cell malignancies. Expert Rev Hematol 2010 Oct;3(5):593-602.

2. Westreich RW, Lawson W. Midline necrotizing nasal lesions: analysis of 18 cases emphasizing radiological and serological findings with algorithms for diagnosis and management. Am J Rhinol 2004 Jul-Aug;18(4):209-19.

3. Rachapalli SM, Kiely PD. Cocaine-induced midline destructive lesions mimicking ENTlimited Wegener's granulomatosis. Scand J Rheumatol 2008 Nov-Dec;37(6):477-80.

4. Wiesner O, Russell KA, Lee AS, Jenne DE, Trimarchi M, Gregori G, Specks U. Antineutrophil cytoplasmic antibodies reacting with human neutrophil elastase as a diagnostic marker for cocaine-induced midline destructive lesions but not autoimmune vasculitis. Artritis Rheum 2004 Sep;50(9):2954-65.

5. Hearing MC, Zink AN, Wickman K. Cocaineinduced adaptations in metabotropic inhibitory signaling in the mesocorticolimbic system. Rev Neurosci 2012;23(4):325-51.

6. Silvestre FJ, Pérez-Herbera A, Puente-Sandoval A, Bagán JV. Hard palate perforation in cocaine abusers: a systematic review. Clin Oral Invest. 2010 Dec;14(6):621-8.

7. Fauci AS, Haynes BF, Katz P, Wolff SM. Wegener's granulomatosis: prospective clinical and therapeutic experience with 85 patients for 21 years. Ann Intern Med 1983 Jan;98(1):76-85.

8. Almouhawis HA, Leao JC, Fedele S, Portes SR. Wegener's granulomatosis: a review of clinical features and an update in diagnosis treatment. $\mathrm{J}$
Oral Pathol Med 2013 Jan 10. doi: 10.1111/jop. 12030. [Epubahead of print].

9. Trimarchi M, Sinico RA, Teggy R, Bussi M, Specks U, Meroni PL. Otorhinolaryngological manifestations in granulomatosis with polyangiitis (Wegener's). Autoinmune Rev 2013 Feb;12(4):501-5.

10. Vega Braga FL, Machado de Carvalho G, Caixeta Guimaräes A, Scaramussa L, Jordao Gusmao R. Otolaryngological manifestations of Wenener's disease. Acta Otorrinolaringol Esp 2013 Jan;64 (1):45-9.

11. Peikert T, Finkielman JD, Hummel AM, McKenney ME, Gregorini G, Trimarchi M, Specks U. Functional characterization of antineutrophil cytoplasmic antibodies in patients with cocaine-induced midline destructive lesions. Artritis Rheumat 2008 May; 58(5):1546-51.

12. Allen CM, Camisa Ch, Salewski C, Weiliand JE. Wegener's granulomatosis: Report of three cases with oral lesions. J Oral Maxillofac Surg 1991; 49:294-8.

13. Kasifoglu T, Cansu D, Koskmaz C. Clinicalimages: perforation of the nasal septum and palate due toWegener's granulomatosis. Artritis Reum 2008 Aug;58(8):2564.

14. Cannady SP, Batra PS, Koening C, Lorenz RR, Citardi MJ, Langford C, Hofmann GS. Sinonasal Wegener granulomatosis: a single-institution experience with 120 cases. Laryngoscope 2009 Apr;119(4):757-61.

15. Silvestre FJ, Salort.Llorca C, Mínguez-Serra MP, Silvestre-Rangil J. Cocaine-related oronasal communication and hard palatede struction. $\mathrm{J}$ Investig Dental 2012 May;3(2):157-60.

\section{CORRESPONDENCIA}

Rafael Crovetto Martínez

Máximo Aguirre, 5, $4^{\circ} \mathrm{B}$

Getxo. Vizcaya. España

E-mail: macdlt@telefonica.net 